\title{
Nanofabrication and Characterization of Plasmonic Structures
}

\author{
Yongqi $\mathrm{Fu}^{1}$, Fengzhou Fang ${ }^{2}$ and Zongwei $\mathrm{Xu}^{2}$ \\ ${ }^{1}$ School of Physical Electronics, \\ University of Electronic Science and Technology of China, \\ ${ }^{2}$ Key Laboratory of Precision Measuring Technology $\mathcal{E}$ Instruments \\ Centre of MicroNano Manufacturing Technology, Tianjin University,
}

China

\section{Introduction}

The nanofabrication processes can be divided into two well defined approaches: 1) 'topdown' and 2) 'bottom-up'. The 'top-down' approach uses traditional methods to guide the synthesis of nanoscale materials. The paradigm proper of its definition generally dictates that in the 'top-down' approach it all begins from a bulk piece of material, which is then gradually or step-by-step removed to form objects in the regime of nanometer-size scale. Well known techniques such as photo lithography, electron beam lithography, anodization, and ion- and plasma-etching, that will be later described, all belong to this type of approach. The top-down approach for nanofabrication is the method firstly suggested by Feynman in his famous American Physical Society lecture in 1959.

Top down fabrication can be likened to sculpting from a block of stone. A piece of the base material is gradually eroded until the desired shape is achieved. That is, you start at the top of the blank piece and work your way down removing material from where it is not required. Nanotechnology techniques for top down fabrication vary but can be split into mechanical and chemical fabrication techniques. The most top down fabrication technique is nanolithography. In this process, required material is protected by a mask and the exposed material is etched away. Depending upon the level of resolution required for features in the final product, etching of the base material can be done chemically using acids or mechanically using ultraviolet light, and x-rays or electron beams. This is the technique applied to the manufacture of computer chips.

Bottom up fabrication can be described as building a brick house. Instead of placing bricks one-by-one at a time to produce a house from bottom, bottom up fabrication technology places atoms or molecules one-by-on at a time to build the desired nanostructure. Such processes are time consuming and so self assembly techniques appeared where the atoms arrange themselves as required. Self assembling nanomachines are regularly mentioned by science fiction writers but significant obstacles including the laws of physics will need to be overcome or circumvented before this becomes a reality. Other areas involving bottom up fabrication are already quite successful. Manufacturing quantum dots by self-assembly 
quantum dots has rendered the top down lithographic approach to semiconductor quantum dot fabrication virtually obsolete. One of the basic 'bottom-up' techniques is chemical precipitation by which nanoparticles of metals, alloys, and oxides etc. are prepared in aqueous or organic solutions. There are several ways to obtain nanoscale precipitates. They can be derived by

1. A controlled phase transformation (i.e., liquid state diffusion) guided by the free energy diagrams or

2. By controlling the solid state diffusion: following a composite route approach mixing, e.g., two different materials and stirring them mechanically.

3. Other approaches can be found in exploiting internal oxidation of materials or thin film deposition of coatings or sputtering.

For fabrication of plasmonic nanostructures, the most commonly used methods are selfassembly of nanoparticles or monomer/polymer molecules and electrochemical deposition (includes electroplating and electro-deposition). This chapter involves both top-down and bottom-up technologies which are being used for fabrication of plasmonic nanostructures and devices.

\section{Nanofabrication of plasmonic structures}

In this chapter, we targeted two commonly used top-down approaches for fabrication of subwavelength metallic structures: focused ion beam (FIB) technology and laser interference photolithography. The former can realized fine nanofabrication over a local tiny area in onestep only. But it is a technique with high expenditure and small localized fabrication area. The latter can realize large area fabrication and cost effective. But it needs pattern transformation from photoresist into substrate. As a typical bottom-up technique, selfassembly monolayer is introduced for nanofabrication of metallic particles array.

Some fabrication examples were presented. Problems existing in the fabrication processes were addressed as well.

\subsection{Focused ion beam technology}

\section{A. Metallic nanoparticles array}

As well known, LSPR-enhanced transmission plays a crucial role for the spectrum during detection. The LSPR effect modifies electromagnetic (EM) fields and indirectly affects the spectrum width which determines detection resolution for biosensing. Therefore, investigating the EM fields is necessary while design the metallic nanoparticles arrays. A few reports were found for design of the metallic nanoaprticles arrays in EM fields analysis points of view. In this paper, we proposed an approach for design of the metallic nanostructure arrays with E-field enhancements through the EM fields calculation. To calculate the EM fields of the metallic nanoaprticles array, we need to consider resonance wavelength $\lambda_{\text {SPP }}$ firstly because the LSPR can only be excited in monochromatic incident light with linear polarization (TM mode for asymmetric structures). However, currently, besides Mie theory for sphere and ellipse particles, there is no theoretical model can be used to directly calculate the resonance wavelength for the metallic nanoparticles array with complicated shapes such as triangular, rhombic, star, and prolate etc. A computational numerical analysis must be employed to calculate the resonant wavelength $\lambda_{\text {spP. }}$. 
Considering this, our approach is that firstly calculate the spectrum (extinction efficiency) of the array using FDTD algorithm. Then peak wavelength of the spectrum is regards as the resonant wavelength $\lambda_{\text {SPP. }}$. After that, we use the resonant wavelength $\lambda_{\text {SPP }}$ as the incident light to calculate the corresponding EM fields of the particles array. In order to fully understand the propagation properties and EM fields distribution, three-dimensional (3D) computational numerical calculation is carried out on the basis of the FDTD algorithm.

Focused ion beam (FIB) direct milling technique has advantages of one-step fabrication process, no material selectivity, and locally tiny area milling. Two-dimensional patterns with arbitrary shapes can be fabricated. Therefore, FIB technique provides large design freedom for the nanoparticles designing that is incomparable for the chemistry-based techniques. The milling experiments were carried out by our FIB machine (FEI Nova 200) with a liquid gallium ion source. This FIB machine is integrated with a scanning electron microscope (SEM) and a gas-assistant etching (GAE) functions. This machine used a focused $\mathrm{Ga}^{+}$ion beam with energy of $30 \mathrm{keV}$, a probe current of $1 \mathrm{pA} \sim 20 \mathrm{nA}$. For the smallest beam currents, the beam was focused down to $7 \mathrm{~nm}$ in diameter at full width and half maximum (FWHM). Using a computer program the milling process is carried out by means of varying the ion dose for different relief depths. The defined area for the FIB with bitmap function and zero-overlap scanning was $1.5 \times 1.5 \mu \mathrm{m}^{2}$. Processing parameters in our FIB experiment is $30 \mathrm{keV}$ ion energy, $10 \mathrm{pA}$ beam current, and $5 \mu$ s dwell time. Considering the inherent ion beam broadening effect, firstly, a bow-tie structure was fabricated using the FIB direct milling for optimization of the process parameters. Figure 1 is the FIB fabricated rhombic particles array. It can be seen that the bow-tie-shaped nanoparticles array was well etched with sharp corners and apexes.

Figure 2 (a) shows the X-Y cross-section and three dimensional (3D) plot of a single crossshaped gold nanoparticle. Along the $x$-axis direction, the distance along the external vertices is denoted as long axis $a$, and the distance along the internal vertices is denoted as short axis $b, h=60 \mathrm{~nm}$. Our previous theoretical study revealed that the nanoparticles with such shape can generate significant SPPs enhanced E-field intensity with coupled "hot-spots" which is a key factor for SERS.

Using a computer program the milling process is carried out by means of varying the ion dose for different relief depths. The defined area for the FIB with bitmap function and zerooverlap scanning was $4 \times 4 \mu \mathrm{m}^{2}$. Processing parameters in our FIB experiment is $30 \mathrm{keV}$ ion energy, $10 \mathrm{pA}$ beam current, and $5 \mu$ s dwell time. Figure 2 (b) is SEM micrograph of the FIB fabricated particles array. It can be seen from the inset image that the adjacent spatial gap is $29.2 \mathrm{~nm}$. The cross-shaped nanoparticles array was well etched with sharp corners and apexes.

A hybrid spider-web-like rhombic nanoparticles array was designed, as shown in Figs. 3 (a) and (b). We adopted this specific pattern based on the following considerations:

1. Ensure interconnections of metallic nanowires for each rhombic particle.

2. Leave extra space for FIB fabrication due to its inherent line broadening effect in horizontal direction, and the web lines themselves having tip features from which "hot spots" can be formed.

3. The lines with two different sizes were designed so as to form irregular web lines in which some lines are broken and the others are connected with the rhombic particles because of the FIB broadening effect. 


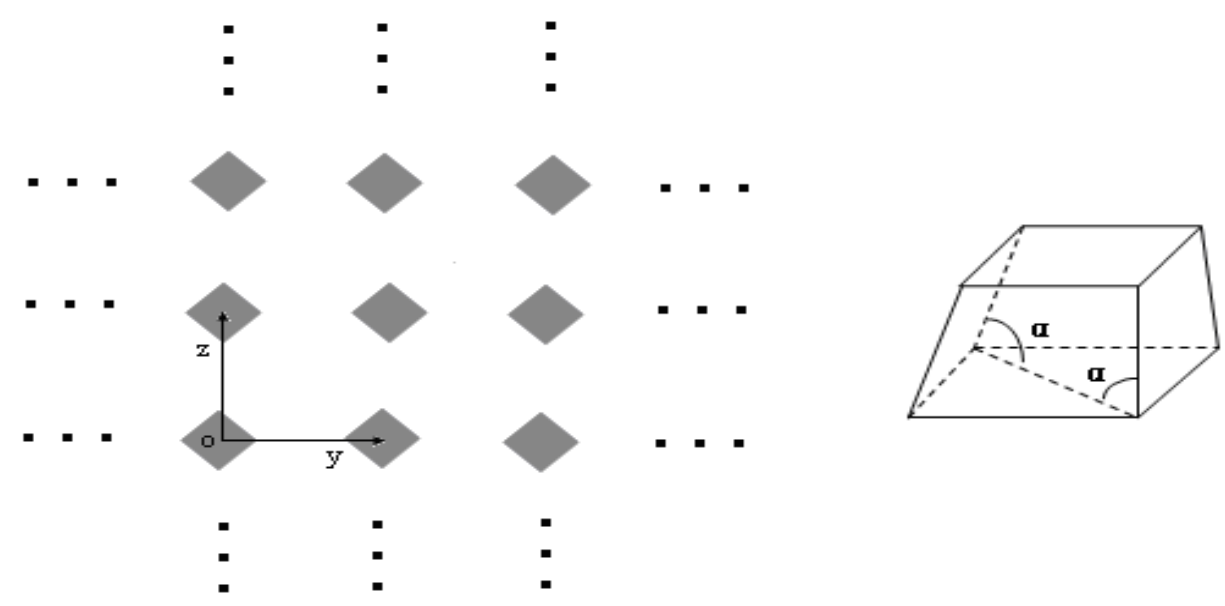

(a)

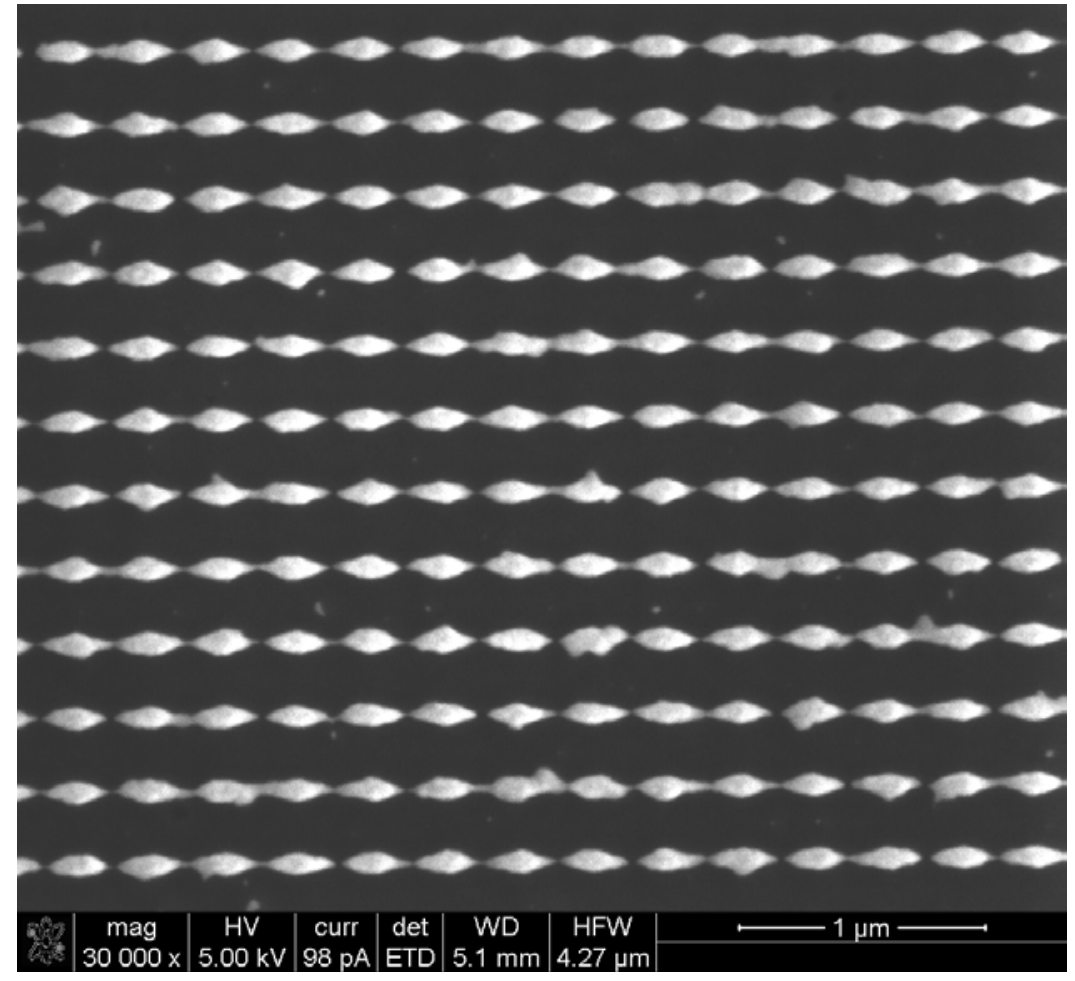

(b)

Fig. 1. (a) Symmetrical two-dimensional infinite rhombic silver nanostructures. (b) SEM micrograph of the FIB fabricated rhombic nanoparticles array. Inset image is zoom-in of the array 


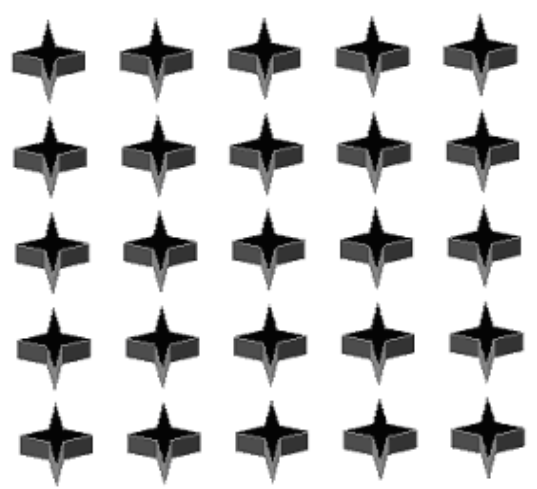

(a)

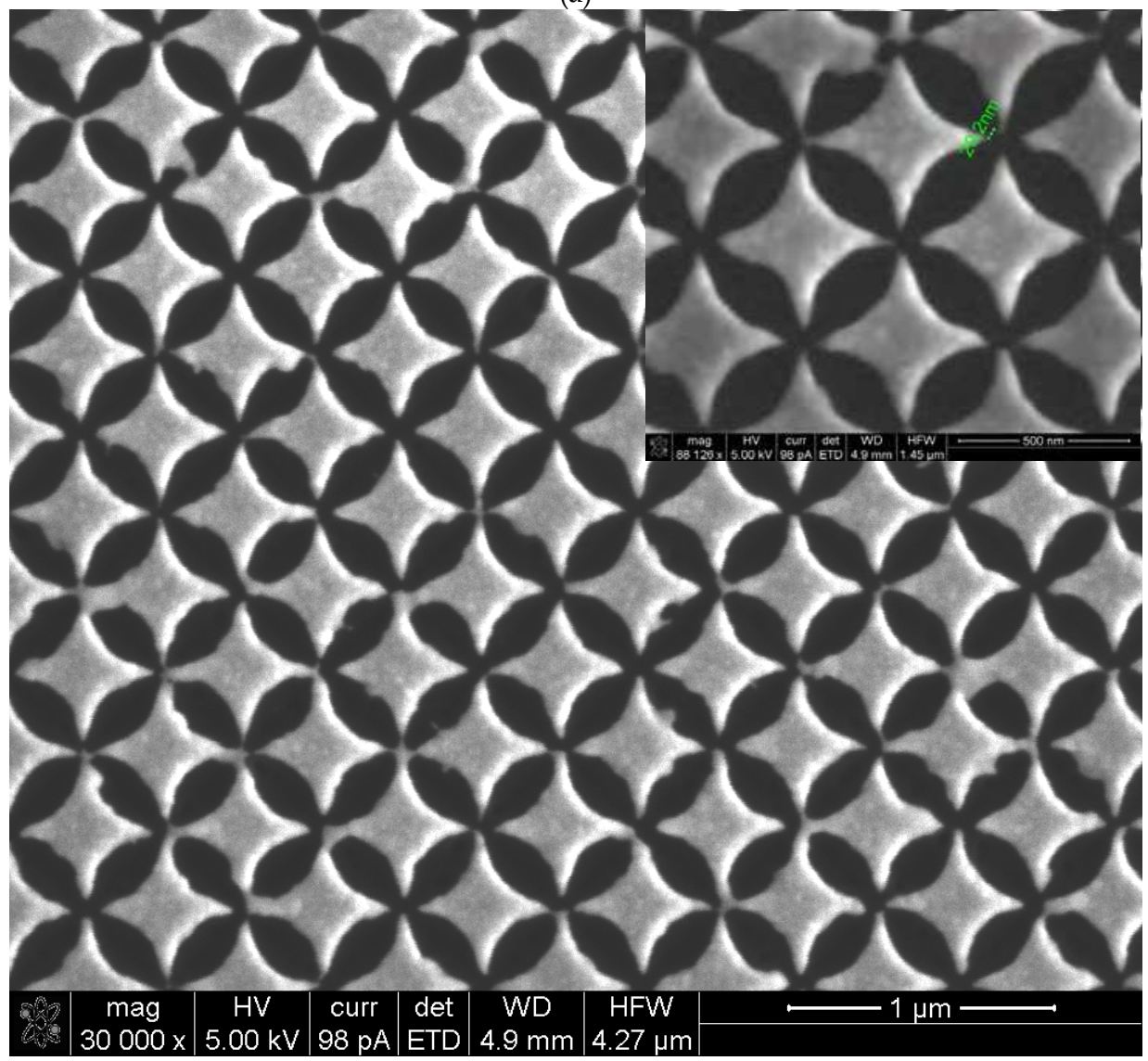

(b)

Fig. 2. (a) Schematic diagram of three-dimensional distribution of the cross-shaped array. (b) Figure 3 SEM micrograph of the FIB fabricated cross-star nanoparticles array. Inset image is zoom in of the array with measured adjacent apex-to-apex space size of $29.2 \mathrm{~nm}$ 
(a)
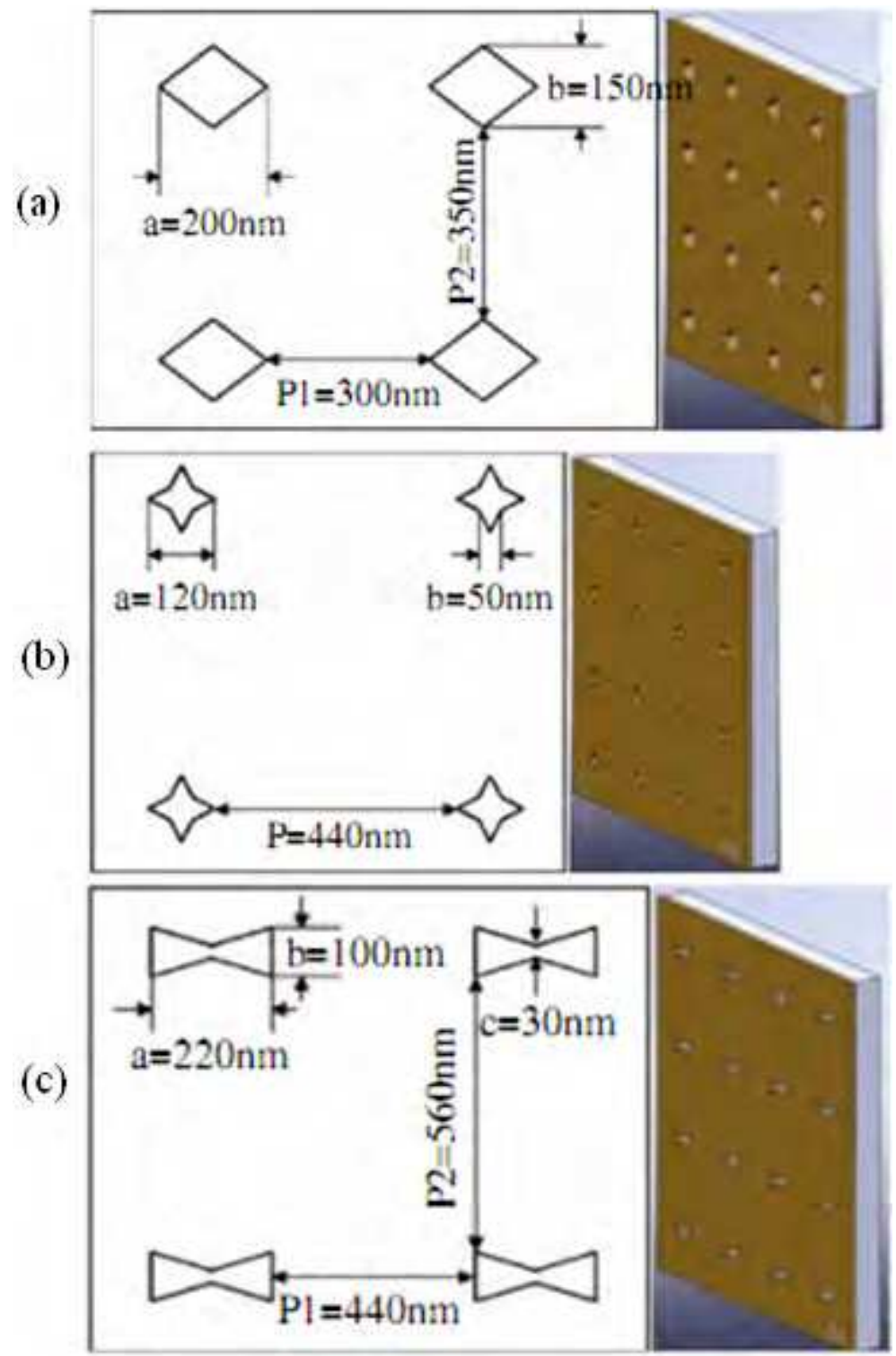

Fig. 3. Schematic drawing of the nanohole arrays with (a) rhombus, (b) cross star, and (c) butterfly nanostructures.

Height of the Au rhombic particles is $60 \mathrm{~nm}$ supported on a glass substrate. The FIB fabricated array area is $20 \times 20 \mu \mathrm{m}^{2}$. Using a computer program the milling process is carried out by means of varying the ion dose for different relief depths. The defined area for the FIB with bitmap function and zero-overlap scanning was $20 \times 20 \mu \mathrm{m}^{2}$. Processing parameters in our FIB experiment is $30 \mathrm{keV}$ ion energy, $10 \mathrm{pA}$ beam current, and $5 \mu$ s dwell time. 


\section{B. Metallic nanoholes array}

Since the extraordinary optical transmission phenomenon was investigated in 1998 [1], nanoholes array have attracted great interest for its unique properties, as well as its potential practical significance for photonic devices and the applications including sensor, optical filtering and lithography [2-10]. The extraordinary optical transmission phenomenon is generally attributed to the excitation of localized Surface Plasmon Polaritons (SPPs) at the edge of the noble metal, and is strongly influenced by the edge shapes of the metal [6]. There are two main surface plasmon modes excited on the metal film. One is the extended surface mode depending on the periodicity; the other is the localized waveguide mode depending on the structure of the hole. The enhanced transmission intensity through the hole is due to the coupling of the two kinds of surface plasmon modes [7, 11]. In comparison to the metallic nanoparticles that behave as radiation, the metallic nanoholes act as surface wave propagation. The extraordinary transmission properties of the cylindrical, rectangular and square hole nanostructure based on simulation and experiment was reported before $[8,12]$. In this chapter, we experimentally investigated the near field optical properties of three different shaped gold nanoholes arrays which were corrugated through an Au film supported on quartz substrate. The near optical transmission properties is obtained using a near-field scanning optical microscope (NSOM), and it is observed that the experimental results are in agreement with the Finite Difference Time Domain (FDTD) calculation results. Using a computer program the milling process is carried out by means of varying the ion dose for different relief depths. The defined area for the FIB with bitmap function and zero-

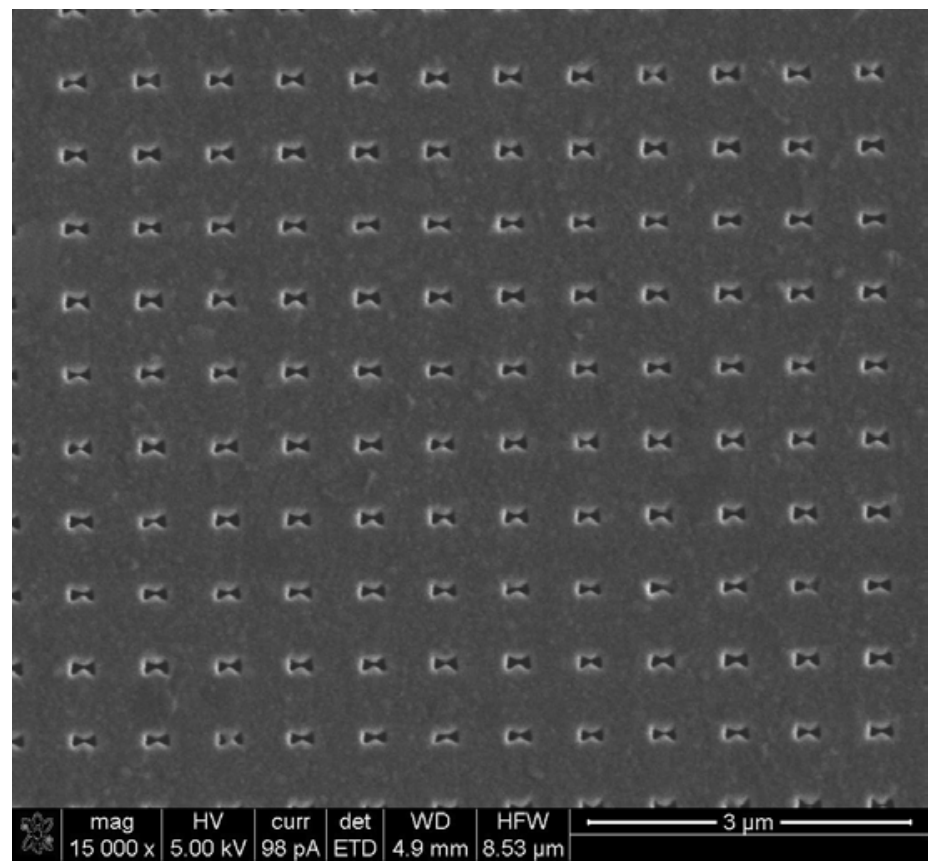

Fig. 4. Scanning electron microscope image of the butterfly nanohole arrays. 


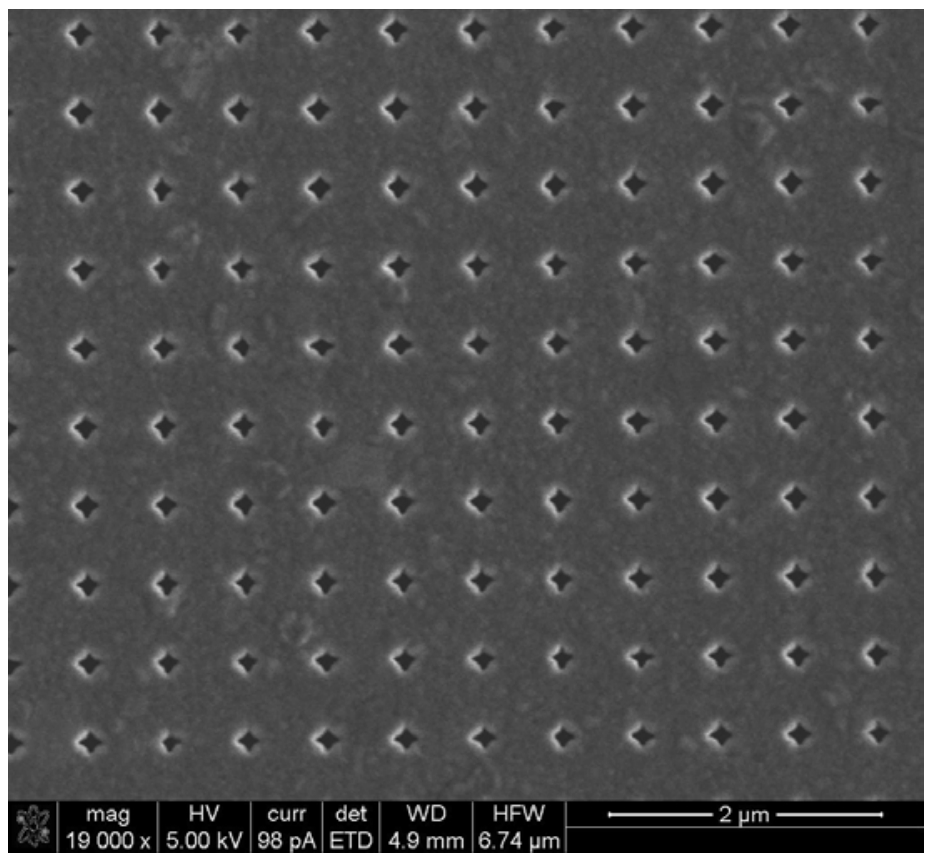

Fig. 5. Scanning electron microscope image of the cross star nanohole arrays.

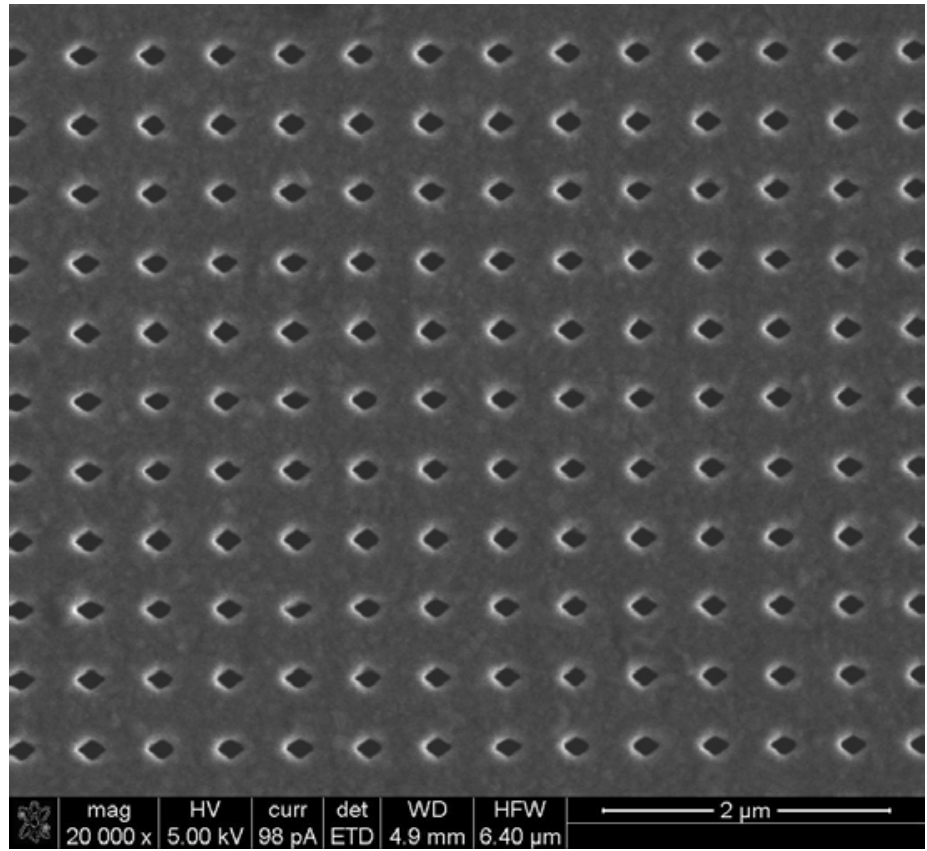

Fig. 6. Scanning electron microscope image of the rhombus nanohole arrays. 
overlap scanning was $25 \times 25 \mu \mathrm{m}^{2}$. Processing parameters in our FIB experiment is $30 \mathrm{keV}$ ion energy, $10 \mathrm{pA}$ beam current, and $5 \mu$ s dwell time. Figure 3 is a schematic drawing of the nanoholes arrays with (a) rhombus, (b) cross star, and (c) butterfly nanostructures. Figure 4, 5 and 6 is SEM micrograph of the FIB fabricated nanoholes array with different shapes of bow-tie, cross-star, and rhombic, respectively.[13]

\section{Plasmonic lenses}

A novel structure called plasmonic micro-zone plate-like (PMZP) or plasmonic lens with chirped slits is put forth to realize superfocusing. It was proposed by our group [14, 15]. Unlike conventional Fresnel zone plate (CFZP), a plasmonic structure was used and combined with a CFZP. Configuration of the PMZP is an asymmetric structure with variant periods in which a thin film of Ag is sandwiched between air and glass. The PMZP is a device that a quartz substrate coated with Ag thin film which is embedded with a zone plate structure with the zone number $\mathrm{N}<10$. Figure 7 (a) is an example of schematic diagram of the structure. Fabrication and characterization of the plasmonic lens was done, as shown in Figs. 7 (a)-(d) [16]. The Au thin film of $200 \mathrm{~nm}$ in thickness was coated on quart substrate using e-beam evaporation technique. The lens was fabricated using focused ion beam (FEI Quanta 200 3D dual beam system) direct milling technique, as shown in Fig. 7 (b). Geometrical characterization was performed using an atomic force microscope (Nanoscope 2000 from DI company). Figure 7 (c) shows topography of the FIB fabricated plasmonic lens. The optical measurement was performed with a near-field optical microscope (MultiView $2000^{\mathrm{TS}}$ from Nanonics Inc. in Israel) where a tapered single mode fiber probe, with an aperture diameter of $100 \mathrm{~nm}$, was used working in collection mode. The fiber tip was raster scanned at a discrete constant height of $500 \mathrm{~nm}, 1.0 \mu \mathrm{m}, 1.5 \mu \mathrm{m}, 2.0 \mu \mathrm{m}, 2.5 \mu \mathrm{m}, 3.0 \mu \mathrm{m}, 3.2$ $\mu \mathrm{m}, 3.5 \mu \mathrm{m}, 3.7 \mu \mathrm{m}, 4 \mu \mathrm{m}, 4.5 \mu \mathrm{m}$, and $5 \mu \mathrm{m}$, respectively, above the sample surface, and allowing us to map the optical intensity distribution over a grid of $256 \times 256$ points spanning an area of $20 \times 20 \mu \mathrm{m}^{2}$. Working wavelength of the light source is $532 \mathrm{~nm}(\mathrm{Nd}$ : YAG laser with power of $20 \mathrm{~mW}$ ).

Additionally, a typical lock-in amplifier and optical chopper were utilized to maximize the signal-to-noise ratio. Figure 7 (d) shows the measured three-dimensional (3D) electric field intensity distribution of the lens at propagation distance of $2.5 \mu \mathrm{m}$.

To further improve focusing quality of the circular holes-based plasmonic lens, an elliptical nanoholes-based plasmonic lens was put forth, as shown in Fig. 8 (a) [17]. The plasmonic lens is composed of elliptical pinholes with different sizes distributed in different rings with variant periods. Long-axis of the ellipse is defined as $a_{n}=3 w_{n}$, whereas $w_{\mathrm{n}}$ is width of the corresponding ring width, and $n$ is the number of rings. A thin film of Ag coated on the glass substrate is perforated by the pinholes. The numbers of $w_{\mathrm{n}}$ and radius for different rings are listed in Table1.

\begin{tabular}{|l|c|c|c|c|c|c|c|c|}
\hline Ring No. & 1 & 3 & 3 & 4 & 5 & 6 & 7 & 8 \\
\hline $\begin{array}{l}\text { Ring } \\
\text { radius }(\mu \mathrm{m})\end{array}$ & 1.41 & 2.11 & 2.78 & 3.43 & 4.08 & 4.73 & 5.37 & 6.01 \\
\hline$w_{\mathrm{n}}(\mathrm{nm})$ & 245 & 155 & 116 & 93 & 78 & 67 & 59 & 53 \\
\hline
\end{tabular}

Table 1 . The numbers of $w_{\mathrm{n}}$ for different rings with orders from inner to outer (designed $f=1 \mu \mathrm{m})$ 

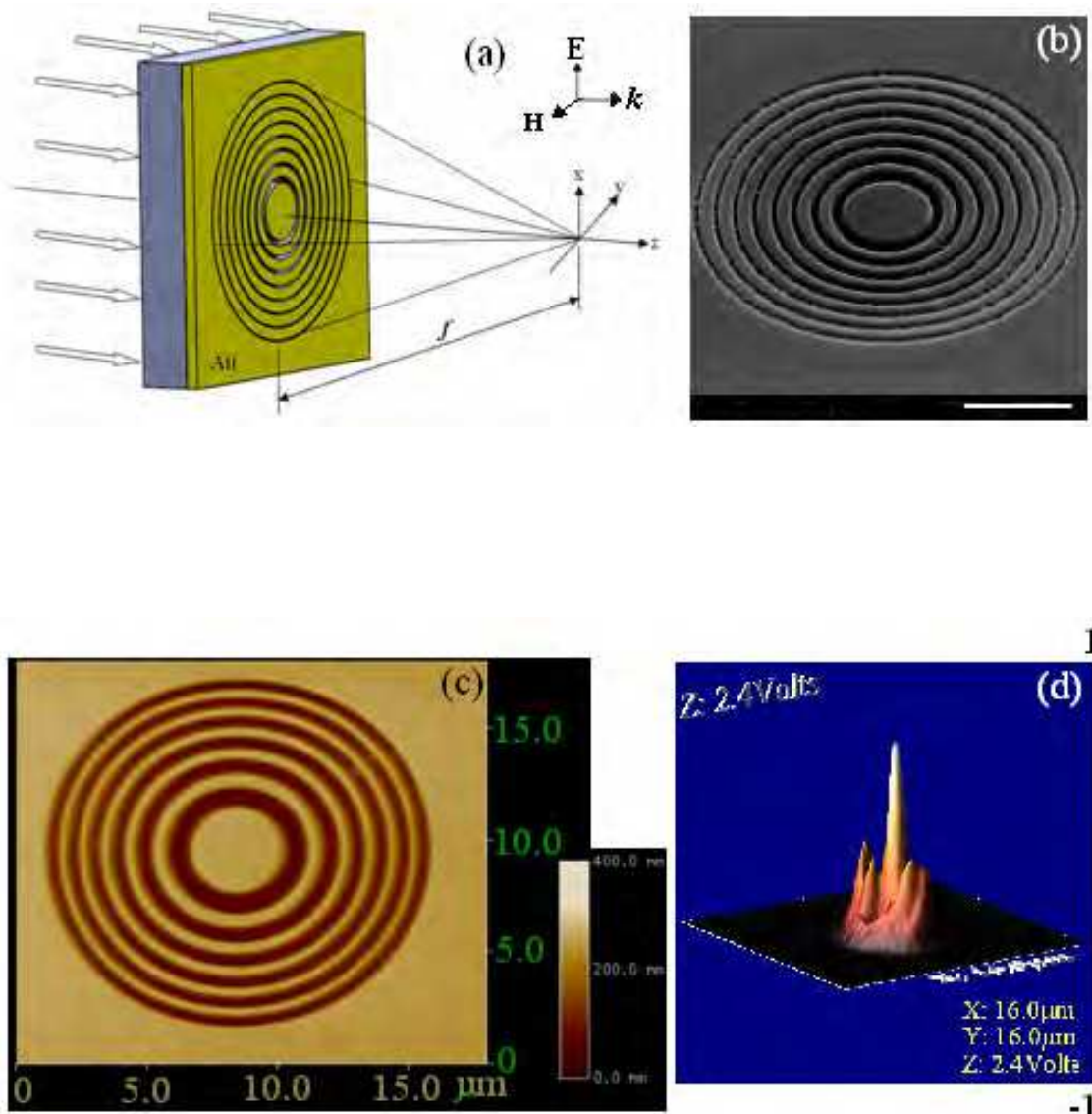

1.22Volts

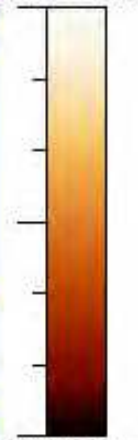

-1.22 Volts

Fig. 7. (a) Schematic diagram of the sandwiched plasmonic lens with chirped circular slits corrugated on Au film. Width of the outmost circular slit is $95 \mathrm{~nm}$. Lens dimension (outer diameter) is $12 \mu \mathrm{m}$. (b) Scanning electron microscope image of the lens fabricated using focused ion beam milling technique. The scale bar is $4 \mu \mathrm{m}$. (c) AFM measurement result: topography of the fabricated lens. (d) NSOM characterization result of the lens: 2D E-field intensity distribution at propagation distance of $2.5 \mu \mathrm{m}$ 


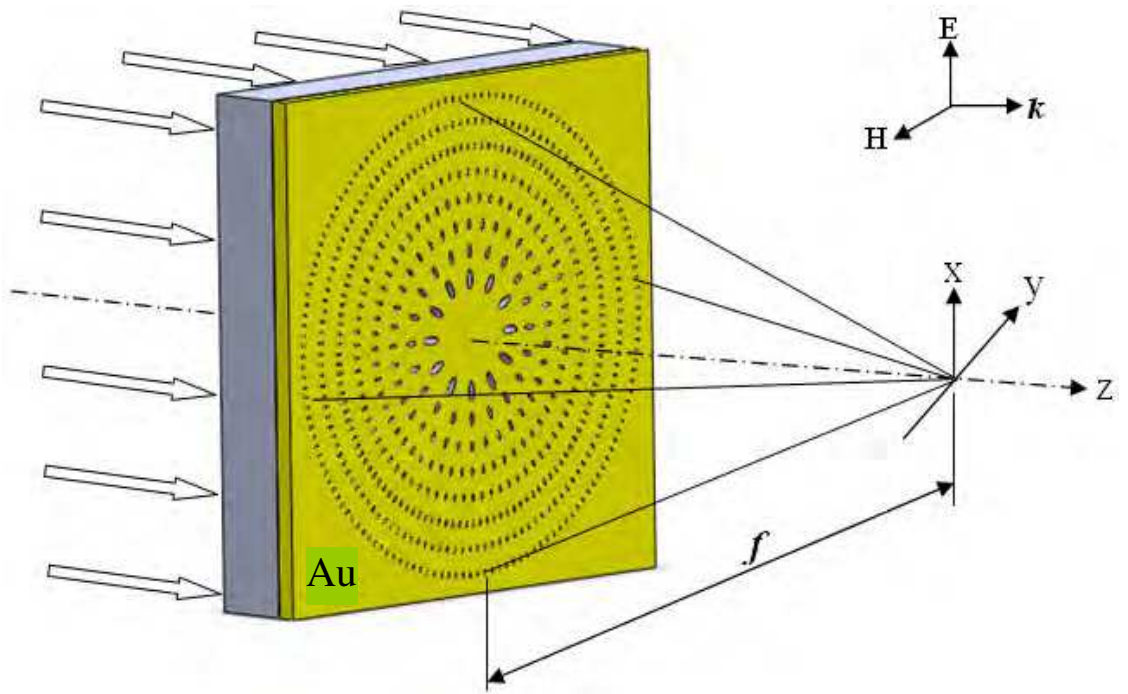

(a)

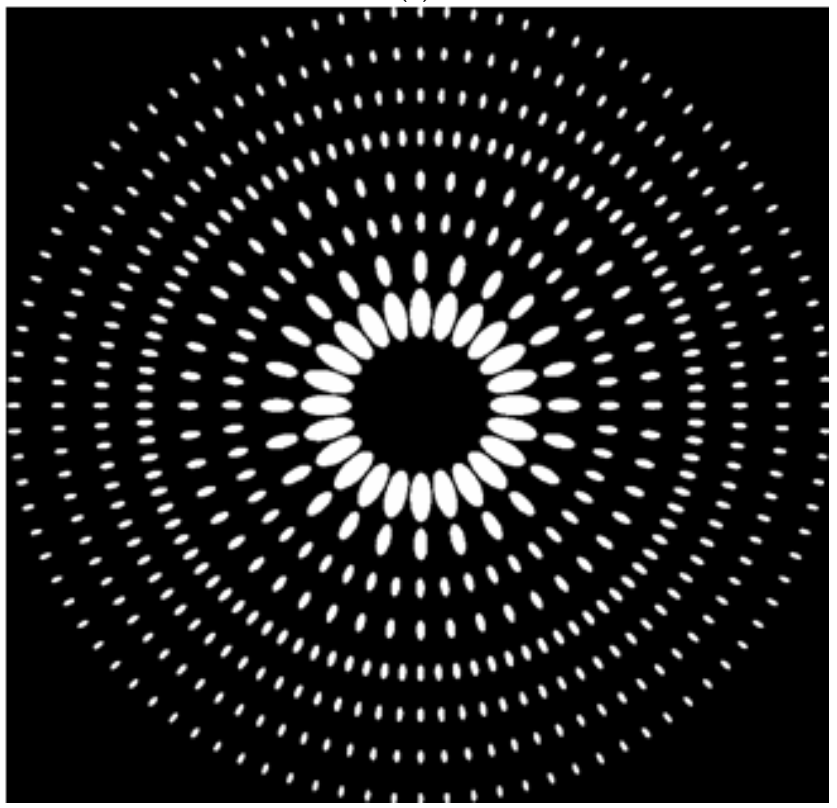

(b)

Fig. 8. (a) Schematization of the pinhole array with focal length $f$. Lateral central distance $L$ determines of wave coupling between the neighbored holes. The pinholes are uniformly distributed along the zones. It is illuminated by various waves with $633 \mathrm{~nm}$ incident wavelength. And we have different polarization states such as TM, EP and RP. (b) layout of elliptical pinholes with total 8 rings $\delta=0.6$ being used in our computational numerical calculation. 


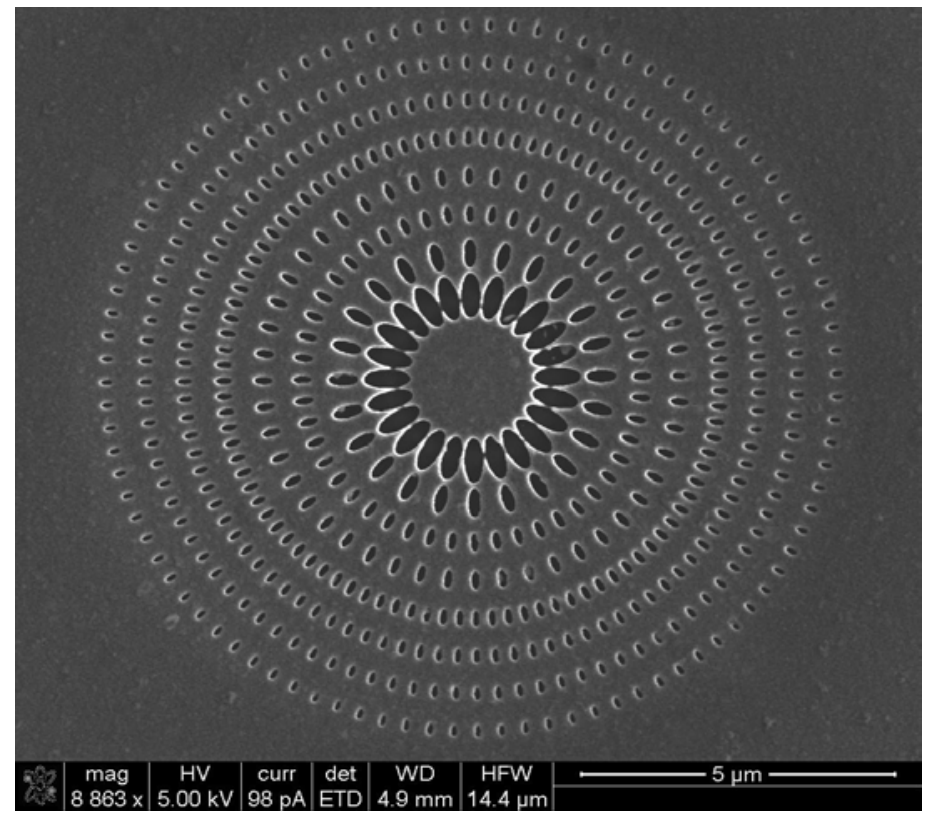

(a)

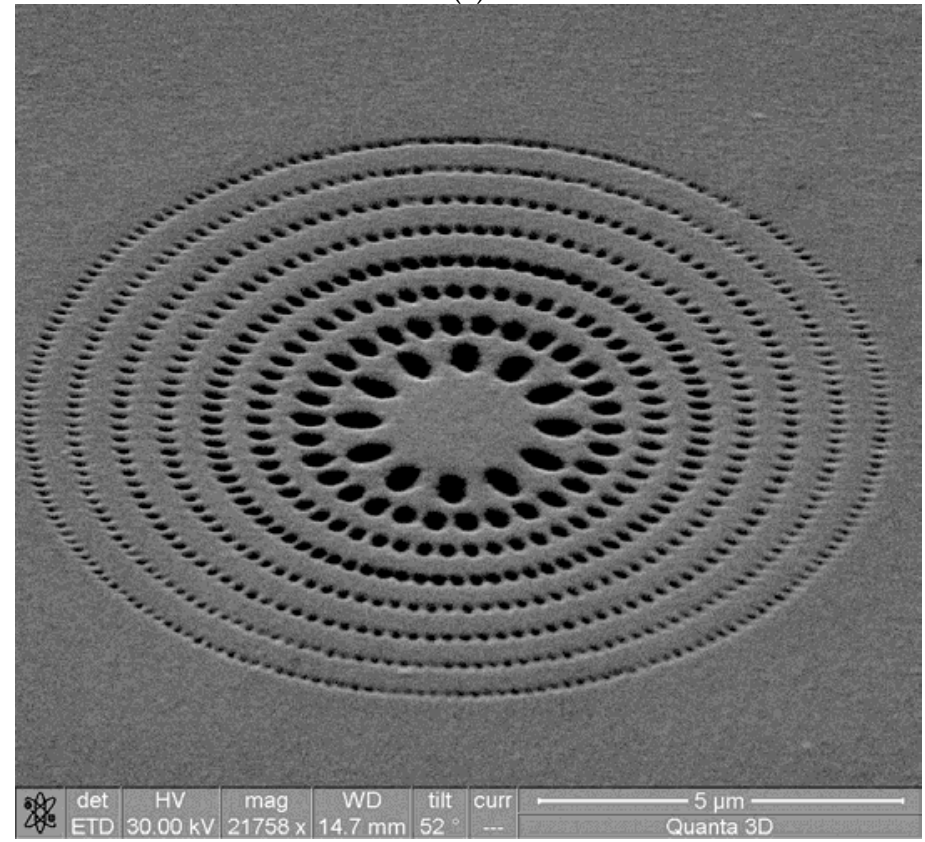

(b)

Fig. 9. (a) Top view of SEM micrograph of the elliptical nanopinholes-based plasmonic lens. (b) Bird view of SEM image of the elliptical nanopinholes. 
As an example, the authors studied the case of $200 \mathrm{~nm}$ thickness Au film coated on quartz substrate and designed a nanostructure with 8 rings on the metal film (see Fig. 8 (b)). The pinholes are completely penetrated through the $\mathrm{Au}$ film. The number of pinholes from inner to outer rings is $8,20,36,55,70,96,107$, and 140 , respectively. Outer diameter of the ring is $12.05 \mu \mathrm{m}$. Radius of the rings can be calculated by the formula $r_{n}^{2}=2 n f \lambda+n^{2} \lambda^{2}$, where $f$ is the focal length for working wavelength of $\lambda=633 \mathrm{~nm}$ which we used in our works. For simplicity, we define a ratio of short-axis to long-axis $\delta=b / a$ (where $a$ is the length of longaxis of the elliptical pinholes, and $b$ the short-axis). The used metal here is Ag with dielectric constant of $\varepsilon_{m}=-17.24+i 0.498$ at $\lambda=633 \mathrm{~nm}$, and $\varepsilon_{\mathrm{d}}=1.243$ for glass. The incident angle $\theta$ is $0^{\circ}$ (normal incidence). In our analysis, we simulated the cases of the ratios: $\delta=0.1,0.2$, and 0.4 , respectively. The ultra-enhanced lasing effect disappears when the ratio $\delta \rightarrow 1$ (circular pinholes). Orientation of the pinholes is along radial direction. The pinholes symmetrically distribute in different rings with variant periods. It can generate ultra-enhanced lasing effect and realize a long focal length in free space accordingly with extraordinarily elongated depth of focus $(D O F)$ of as long as $13 \mu \mathrm{m}$ under illumination of plane wave in linear $\mathrm{y}$ polarization.

The lens was fabricated using focused ion beam directly milling technique using beam current of $10 \mathrm{pA}$ and ion energy of $30 \mathrm{keV}$, as shown in Figs. 9 (a) and (b).[18]

\subsection{Laser interference photolithography}

A Lloyd's mirror interferometer system was built, as shown in Fig. 10. [19] Light source is He-Cd laser with $442 \mathrm{~nm}$ working wavelength. The laser beam is filtered and expanded by a spatial filter which is composed of Lens 1 and a pinhole. The expanded beam is collimated by Lens 2 . A part of the incident beam is reflected back by the mirror which is positioned in normal to the substrate and interferes with the other non-reflection beam to form the interference patterns. Since the beam is only split with a short path length near the substrate, this setup is very insensitive to the mechanical vibration caused instabilities. Hence no extra feedback control system is required to stabilize the interference fringe patterns [20]. Exposure time is controlled by a shutter. If the light intensity of the collimated beam is $I_{0}$, the radiation on the surface is given by

$$
I_{2-\text { beam }}=2 I_{0}[1+\cos (2 k x \sin \theta)]
$$

where $k=2 \pi / \lambda$, here $\theta$ is the half angle between the two beams. The grating period $d$ is given by

$$
d=\lambda /(2 \sin \theta)
$$

After the first time exposure, the substrate is rotated over $90^{\circ}$ and exposed again with the same exposure time. The distributed intensity on the surface is written as

$$
I_{\text {double }-2 b}=2 I_{0}[2+\cos (2 k x \sin \theta)+\cos (2 k y \sin \theta)]
$$

The period of the interference pattern generated by this method can be easily tuned by means of changing the incidence angle on the mirror as well as wavelength of the light source. 


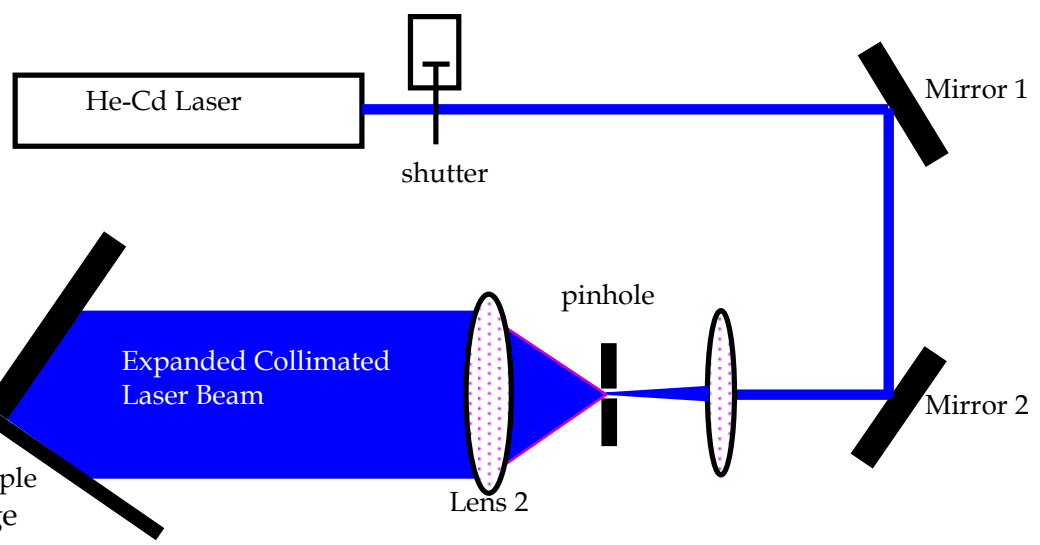

Fig. 10. Experimental setup of the Lloyd's mirror laser interference lithography

The photoresist was exposed by the collimated beam from the Lloyd's mirror interferometer system. A 1D grating pattern of the photoresist layer was formed after the first time exposure and development. Exposure dose was measured to be $1.5 \mathrm{~mW} / \mathrm{cm}^{2}$ in normal incidence. The developer adopted in our experiments is AR 300-35 (Allresist Co.). AFM measurement results after the exposure can be seen in Fig. 11 (a). In order to obtain a 2D grid pattern with the dots array in different sizes on the resist, second exposure with varied exposure time can be performed. The diameter of the holes in the photoresist depends on the exposure time. The dependence gives a possible tool to tune the microhole size at a constant space. Corresponding AFM micrograph is shown in Fig. 11 (b) and (c) respectively. In order to analyze the pattern period, an AFM measurement was carried out, which shows that the period is $\sim 400 \mathrm{~nm}$.

\subsection{Self-assembly monolayer}

An extended nanosphere lithography (NSL) technique was used to create the surfaceconfined rhombic Ag nanoparticles supported on a glass substrate, as shown in Fig. 12 (a).[21] Figure 13 shows the fabrication process of the nanoparticles. Firstly, the glass substrate was cleaned. Then the self-assembly of size-monodisperse (the sphere size of the chemical solutions which is spin-coated as a monolayer in which the sphere size determines the generated rhombic particle size.) polystyrene nanospheres (500 nm, 2\%), glass nanospheres (200 nm, 1\%), and distilled water were mixed in 5:1:50 and applied to form a monolayer (see Fig. 12 (b)), and followed by hydrofluoric acid to etch off the glass nanospheres, and PS nanospheres are remained on the substrate (see Fig. 13 (c)). After that, the Ag particles were deposited through the nanosphere masks using thermal or electron beam evaporation technique. After removal of the polystyrene nanospheres by sonication in absolute ethanol for $3 \mathrm{~min}$., well ordered two-dimensional rhombic nanoparticle arrays were finally obtained on the substrates. By changing the nanosphere diameter and the deposited metal thickness, the nanoparticles with different in-plane width, out-of-plane height and interparticle space can be derived. 


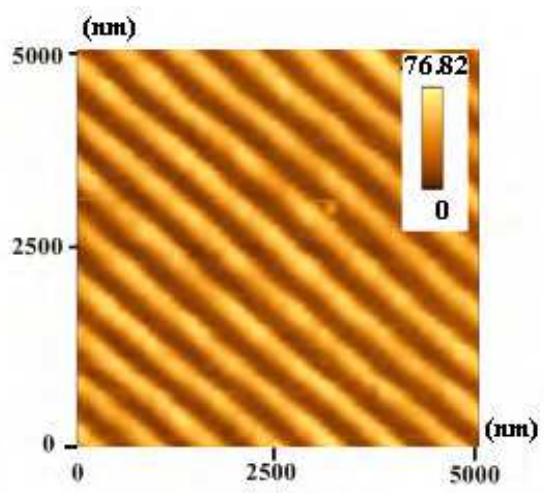

(a)

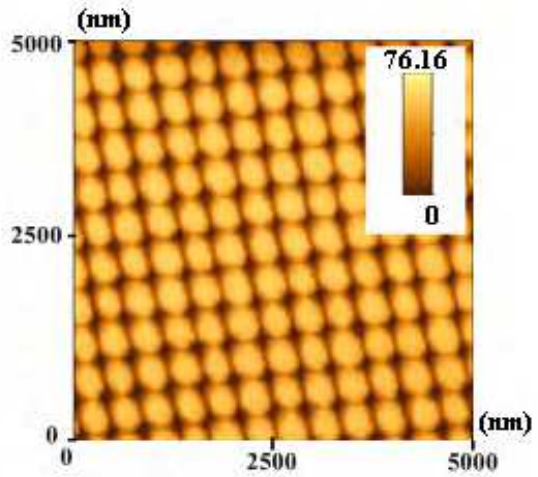

(b)

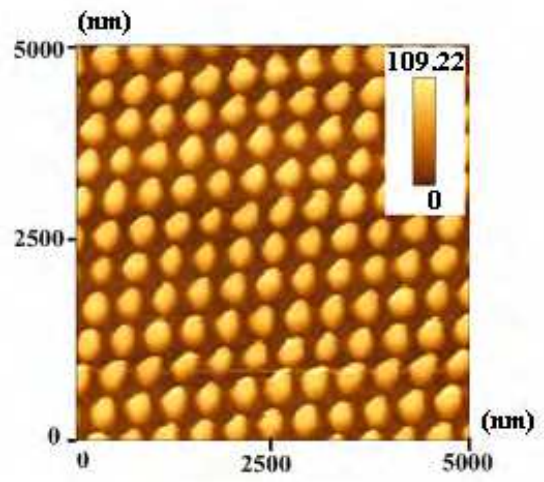

(c)

Fig. 11. AFM micrograph of the photoresist layer that remains after exposure and development in the laser interference setup (a) 1D grating structure after first exposure, (b) 2D grid pattern after second exposure with short exposure time of 8 s., (c) 2D dots array after second exposure with longer exposure time of $13 \mathrm{~s}$. 


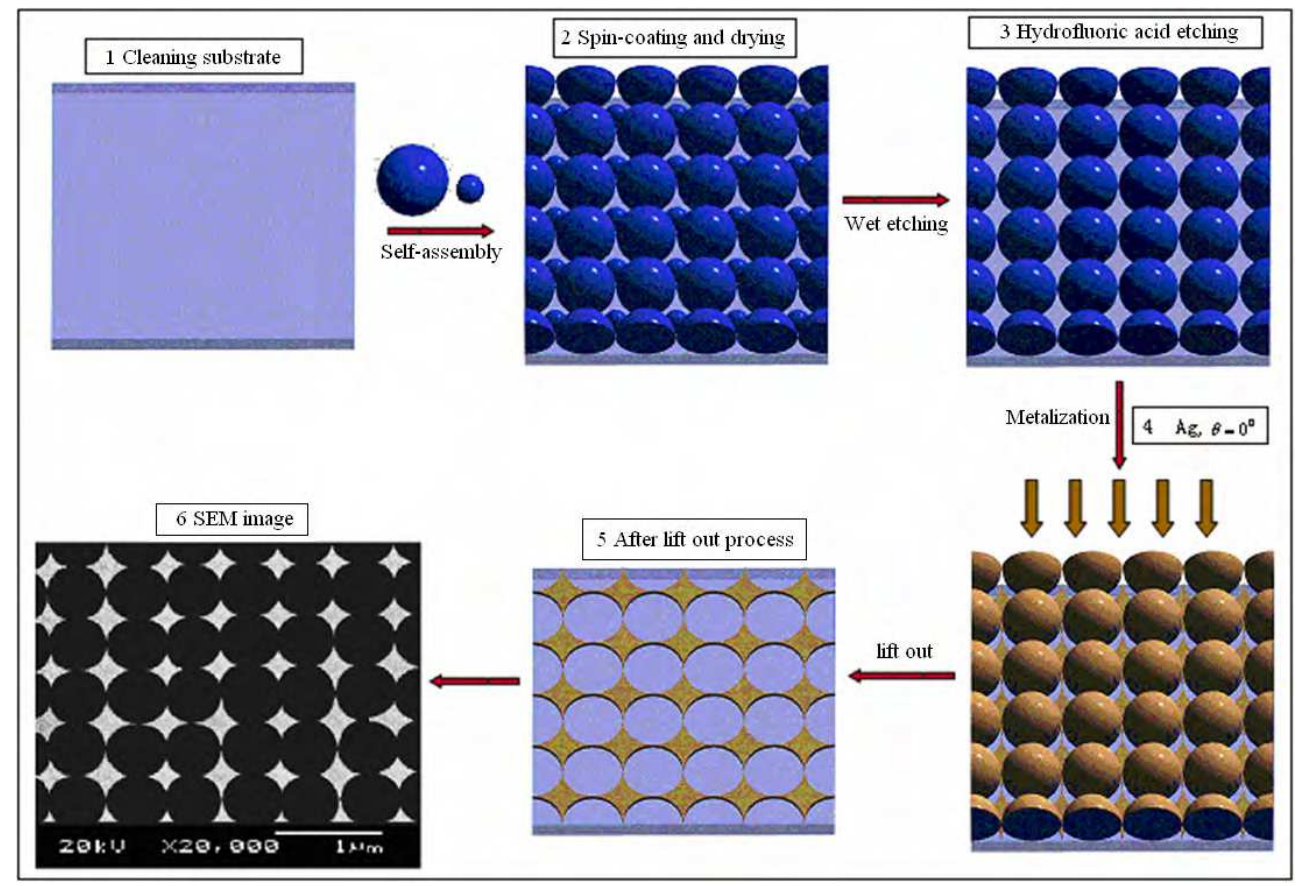

(a)

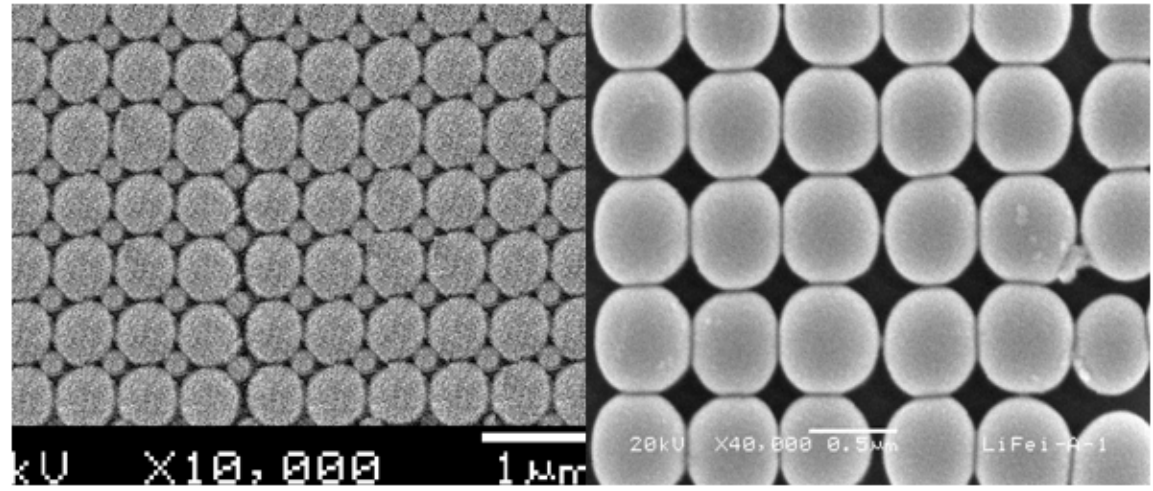

(b)

(c)

Fig. 12. (a) The progress of the nanoparticle preparation. (1) glass substrates cleaned, (2) monodisperse polystyrene nanospheres and glass nanospheres were drop coated and dried, (3) hydrofluoric acid was used to etch off the glass nanospheres, (4) Ag metal was vapor deposited onto the sample, (5) lift out the polystyrene nanospheres, (6) representative scanning electron microscope image of substrate. Micrographs of SEM for (b) monolayer consisted of PS nanospheres and glass nanospheres; (c) polystyrene nanospheres being used as a template for next step: Ag metallization. 


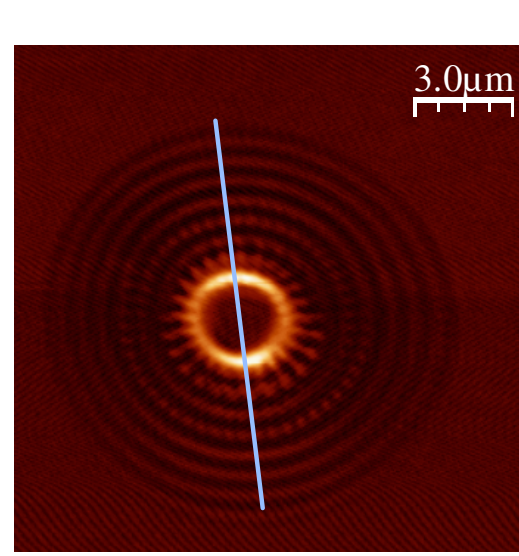

(a)

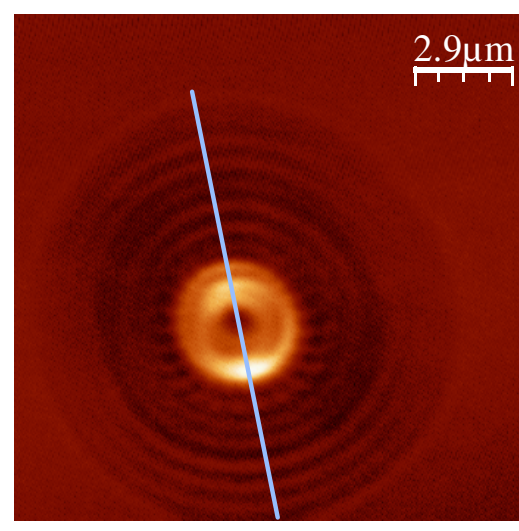

(c)

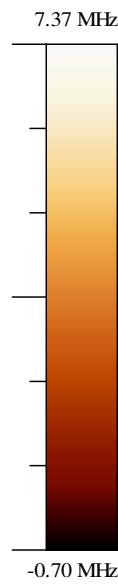

$-0.70 \mathrm{MH}$

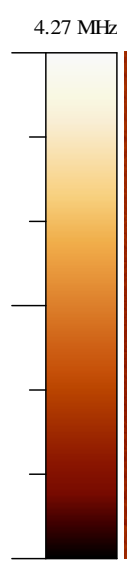

$-0.71 \mathrm{MHz}$

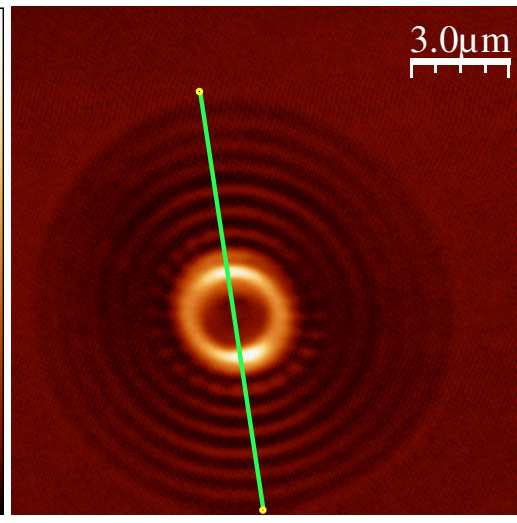

(b)
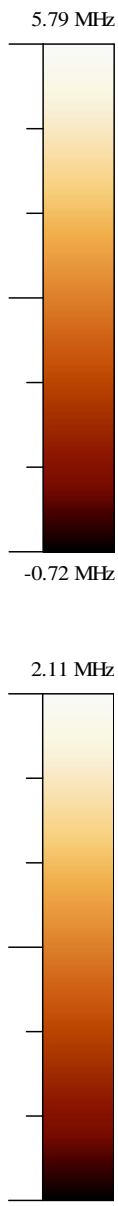

$-0.60 \mathrm{MHz}$

(d)

Fig. 13. NSOM probed E-field intensity distribution along different propagation distance $Z$ (shown as the lines in the images), working in sample scan mode. (a) $Z=10 \mathrm{~nm}$; (b) $Z=0.5$ $\mu \mathrm{m}$; (c) $Z=1.5 \mu \mathrm{m}$; (d) $Z=2.0 \mu \mathrm{m}$. The straight lines here indicate positions of cross-section profiles.

We measured the surface modality of the samples by using JSM-5900LV Scanning Electron Microscope (SEM). The Ag nanorhombus have in-plane widths of $\sim 140 \mathrm{~nm}$ as measured by the SEM and out-of-plane heights of $\sim 40 \mathrm{~nm}$ as measured by sidestep apparatus. We can see that the size of the Ag rhombic nanoparticles fabricated is not identical as the limit of the SAM method, which accounts for the errors in our following experiment.

The fabricated Ag rhombic nanoparticles have larger surface area than that of the Ag triangular nanoparticles [16] when we used the same diameter polystyrene spheres to fabricate them. The surface area of Ag rhombic nanoparticles is 2.5 times larger than the triangular structure. As mentioned above, Ag rhombic nanoparticles lead to a stronger 
enhancement of the localized surface plasmon resonance, and thus result in the improvement of the detection sensitivity and efficiency of the binding reaction between the antigen and antibody.

\section{Characterization of plasmonic structures}

Normally, characterization of plasmonic nanostructures and nanophotonic devices includes geometrical and optical characterizations. Currently, scanning probe microscope (SPM) is a key approach for the geometrical characterization. SPM is a general name for the probe scanning technology-based point-to-point measurement method. It involves atomic force microscope (AFM), scanning tunneling microscope (STM), and near-field scanning optical microscope (NSOM) or scanning near-field optical microscope (SNOM). Amongst, AFM is a most commonly used tool for the geometrical characterization. Optical characterization technology includes NSOM, surface enhanced Raman spectroscopy (SERS), confocal microscopy, and multiphoton microscopy. NSOM and SERS are the most popular tools for the optical characterization. The following sections will describe them one-by-one in detail combining with corresponding experimental results of the relevant plasmonic structures and devices.

\subsection{Near-field scanning optical microscopy}

A near-field scanning optical microscope (NSOM) has been adopted for the purpose of evaluating optical performance of the lens. The theoretical calculation-based analyses were completed for the plasmonic lens consisting of the Au film in Ref. [16]. To obtain the field distribution across planes of different heights slightly above the structure, we used NSOM (MutiView2000TS, from Nanonics Inc.) working in collection mode, namely, the lens is excited by laser beam from far-field and the transmitted light is detected by the fiber probe in near-field region. Light source is a pumped YAG laser with $532 \mathrm{~nm}$ working wavelength. The light is focused to a spot at the surface of the lens through an optical microsope. Thus it is strong enough to be detected at surface of the exit side of the lens. We manually set height of the probe so as to keep the probe scans at a constant working distance. The NSOM probe used in this experiment is $200 \mathrm{~nm}$ in diameter. As a consequence, image resolution of the AFM scanning is limited especially for probing the topography of the FIB fabricated structure. But the image is still clear, as shown in Fig. 7 (b). Considering the radius of the lens and resolution limits, scanning region was defined as $15 \times 15 \mu \mathrm{m}$, and 256 points per scan line. Both tip scan mode and sample scan (moving stage) modes were used to probe the lens respectively.

In our experimental study, we found that the two scan modes, fiber tip/probe scan and sample scan of the NSOM system have different characteristics for probing our plasmonic lenses. Our NSOM system illuminates the sample with Gaussian beam from laser source through an objective lens $(10 \times$, and $N A=0.25)$. For the tip scan mode, the focused beam region is formed in free space already once the incident Gaussian beam illuminates the pinholes structure. Meanwhile, the fiber probe collects the optical signal originating from the whole structure of the lens while scans after exit plane of the lens in free space. Thus in this case, it functions like a dark-field optical microscope to pick-up the optical signal from dark background. But for the sample scan mode, the sample moves together with the stage for scanning while the fiber probe is fixed to collect optical signal transmitted from the lens. 
It means that the whole structured lens area can be fully illuminated by the Gaussian beam. The sample moves relevant to the probe step-by-step or point-by-point. Therefore, central spot of the Gaussian beam at each scan position will be modulated by the pinholes structure while the sample and stage driven by the step-motors. The final scan image will be like an image from a bright-field optical microscope with uniform illumination. Thus the signal intensity collected by the probe is higher for the sample scan mode in comparison to that of the tip scan mode. Considering these features, we can use sample scan for probing transmission property of the lens and tip scan for characterization of focusing performance.

The experiment was carried out for the purpose of revealing focusing phenomenon of the novel lens. Firstly, we verified the focusing effect using NSOM with sample scan mode to collect focusing signal of the incident light from the lens. Figures 13 (a)-(d) are NSOM probed E-field intensity distributions along different propagation distances at $\mathrm{z}=10 \mathrm{~nm}, 0.5$ $\mu \mathrm{m}, 1 \mu \mathrm{m}$, and $2 \mu \mathrm{m}$, respectively. As can be seen, a clear near-field image was obtained at $\mathrm{z}=10 \mathrm{~nm}$ (see Fig. 13 (a)). A focusing area is gradually formed at $\mathrm{z}=2 \mu \mathrm{m}$ (see Fig. 13 (d)).

It is worthy to pint that for the near-field characterization of the plasmonic structures using NSOM, coupling between optical fiber probe and metallic surface of the plasmonic structures will play a negative role. The coupling directly influences accuracy of the NSOM probed near-field intensity distribution. A possible approach to solve the problem is that use near-field phase measurement by means of interference. Then we use the measured nearfield phase distribution to restore the corresponding intensity distribution which is the real result without influence of the coupling on the real intensity distribution because the coupling does not influence the phase distribution.

\subsection{Spectrometry of metallic nanoparticles and nanoholes \\ 3.2.1 Optical spectroscopy}

In order to observe the sensor characteristic of this kind of biochips, the extinction spectrum was measured by a Sciencetech 9055 spectrophotometer. To obtain Visible-IR transmittance spectrum, a white light source $(400 \sim 700 \mathrm{~nm})$ was employed in the experimental system. Incident light was transmitted through a multimode optical fiber and reached collimating lens firstly, and then illuminated onto the biochip. The transmitted light beam through the sample was collected with an identical focus lens which is attached to the multimode fiber. Then a monochromator was used to separate the light from the multimode fiber to be monochromatic light beam. After that, the signal was sent to a personal computer which is integrated with an analog photomultiplier. The transmittance spectra were directly displayed on the screen of the computer. The measuring process was divided into three steps:

1. Measuring of the background light without any input sources and samples;

2. Measuring the light source intensity;

3. Collecting the transmitted light with the presence of the sample which is placed perpendicular to the incident light.

The transmittance $T$ can be written as $T=(s-b) /(r-b)$, where $s, r$, and $b$ denote the intensity of the sample, reference, and background, respectively. The extinction spectra $E$ for each step were achieved by equation $E=-\log T$. In addition, the transmittance spectra can be directly plotted and displayed in computer without further data processing. 
The LSPR spectra of the Ag nanochips in each processing step is presented in Fig. 14 with incidence wavelength ranging from $400 \mathrm{~nm}$ to $700 \mathrm{~nm}$, the resulting extinction spectrum of the bare Ag nanoparticles is depicted in Fig. 14 case a, where the LSPR $\lambda_{\max }$ was measured to be $558.5 \mathrm{~nm}$. Similarly, Fig. 14 case b shows the extinction spectrum after modification of the Ag nanoparticles with $1 \mathrm{mM}$ 1:3 11-MUA/1-OT. To ensure a well-ordered SAM on the Ag nanoparticles, the sample was incubated in the thiol solution for $24 \mathrm{~h}$. After carefully rinsing and thoroughly drying with $\mathrm{N}_{2}$ gas, the corresponding peak transmission with LSPR wavelength $\lambda_{\max }$ was measured to be $572 \mathrm{~nm}$. Compared to the bare Ag nanoparticles, $\lambda_{\max }$ in this surface functionalization step is red-shifted by approximately $13.5 \mathrm{~nm}$. Next, $1 \mathrm{mM}$ biotin was covalently attached via amide bond formation with a two-unit poly (ethylene glycol) linker to the carboxylated surface sites. Obtained LSPR spectrum is indicated in Fig. 14 case c, which shows that the peak transmission occurs at site of $594.5 \mathrm{~nm}$. It corresponds to an additional $22.5 \mathrm{~nm}$ redshift from the second peak (red line). We finally plotted the extinction spectrum in Figs. 14 after the reaction between $100 \mathrm{nM}$ streptavidin and $1 \mathrm{mM}$ biotin. It is found that the maximum wavelength have a $16.5 \mathrm{~nm}$ redshift from $594.5 \mathrm{~nm}$ up to $611 \mathrm{~nm}$. In contrast, using the same diameter PS spheres, the uniform height of the Ag nanoparticles, under the same metrical condition and processing time, we fabricated the

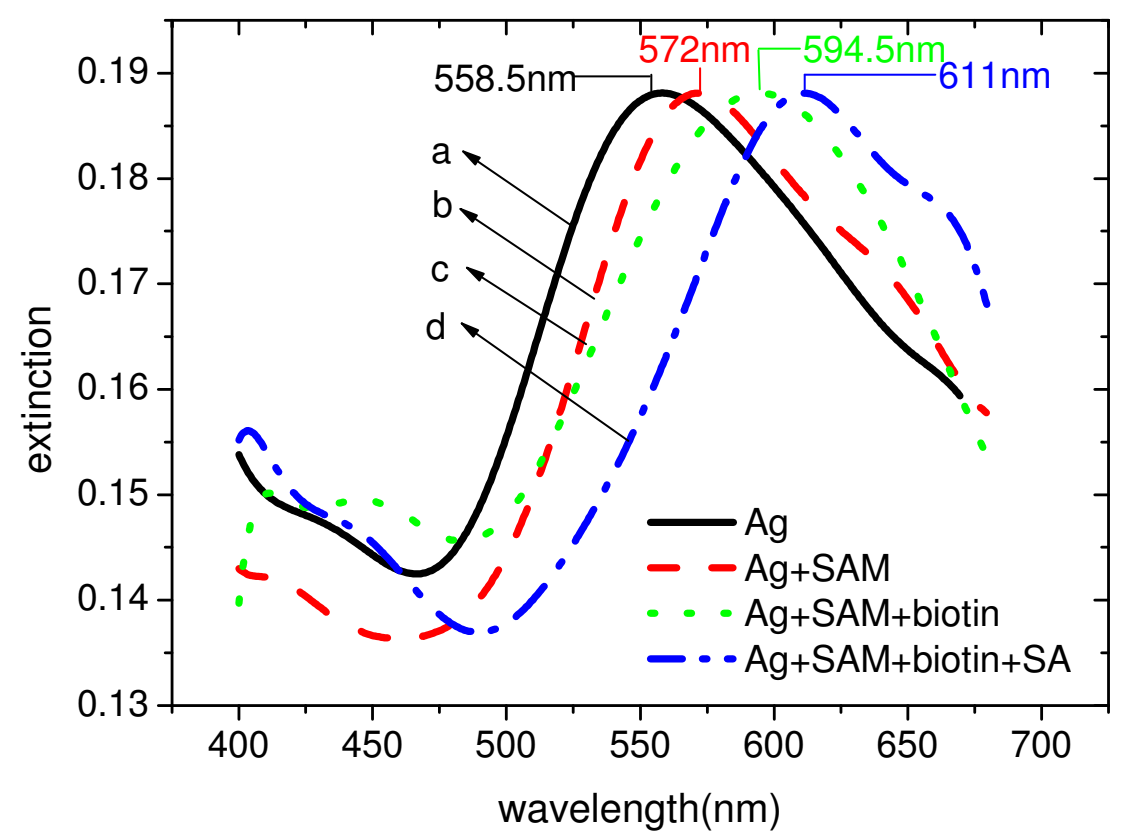

Fig. 14. LSPR spectra of each step in the surface modification of NSL-derived Ag nanoparticles to form a biotinylated Ag nanobiosensor and the specific binding of SA. (a) Ag nanoparticles before chemical modification, $\lambda_{\max }=558.5 \mathrm{~nm}$. (b) Ag nanoparticles after modification with $1 \mathrm{mM}$ 1:3 11-MUA/1-OT, $\lambda_{\max }=572 \mathrm{~nm}$. (c) Ag nanoparticles after modification with $1 \mathrm{mM}$ biotin, $\lambda_{\max }=594.5 \mathrm{~nm}$. (d) Ag nanoparticles after modification with $100 \mathrm{nM} \mathrm{SA}, \lambda_{\max }=611 \mathrm{~nm}$. All extinction measurements were collected in air environment. 
traditional Ag triangular nanosensor, and measured the extinction spectrum. However, in our experiments, for the traditional Ag triangular nanosensor, a $7.5 \mathrm{~nm}$ redshift in the reaction between $100 \mathrm{nM} \mathrm{SA}$ and $1 \mathrm{mM}$ biotin is observed. The shift of $16.5 \mathrm{~nm}$ mainly attributed to the "hot spot" of the rhombic particles mentioned before which plays a dominant role for improving detection sensitivity here.

By analyzing the obtained results, we apparently found that the extinction spectrum varies with each step of the surface functionalization for the Ag nanoparticles. The spectral shifting in those cases can be explained by the change of the local effective refractive index originating from the surface modification in each step.

\subsubsection{Surface enhanced Raman spectroscopy}

After nanofabrication, the sample was cleaned using deionized water. Then two samples were dipped with time of $24 \mathrm{~h}$ and $36 \mathrm{~h}$ respectively in the chemical solution of Rhodamine 6G with concentration of $10 \mu \mathrm{M} / \mathrm{L}$. A Raman spectroscopy with model of Renishaw Invia 2000 from UK was employed for Raman spectrum measurement. Working wavelength of laser source $\left(\mathrm{Ar}^{+}\right.$laser with power of $\left.10 \mathrm{~mW}\right)$ is $800 \mathrm{~nm}$.

Figure 15 (a) is measured Raman spectra of the rhombic nanoparticles array with SERS enhancement for the samples were dipped in $10 \mu \mathrm{Ml} / \mathrm{L}$ Rhodamine 6G. In order to verify influence of the dipping time on Raman intensity and SERS enhancement, we prepared two samples which were dipped in the Rhodamine 6G with different time of $24 \mathrm{~h}$ and $36 \mathrm{~h}$, respectively. Then they were cleaned using deionized water. The following conclusions regarding rhombic particles can be drawn in terms of the Raman spectra:

1. The rhombic particles array can distinguish the peaks of Raman spectrum with apparent positions at $611 \mathrm{~cm}^{-1}, 1184 \mathrm{~cm}^{-1}, 1310 \mathrm{~cm}^{-1}, 1360 \mathrm{~cm}^{-1}, 1509 \mathrm{~cm}^{-1}, 1597 \mathrm{~cm}^{-1}$, and $1650 \mathrm{~cm}^{-1}$, respectively.

2. Background noise exists in the measured SERS signal. It may attribute to the purity of chemical solution and defects from ambient while tests the samples. But the drawbacks caused influence on SERS enhancement is a little.

3. Considering that the samples were cleaned using deionized water, remained Rhodamine $6 \mathrm{G}$ molecule are far lower than the previous concentration of $10 \mu \mathrm{Ml} / \mathrm{L}$. It is reasonable to believe that sensitivity of the rhombic particles is high for the SERS enhancement-based detection.

4. Dipping time is relevant to the Raman spectrum. It can affect both Raman intensity and peak position/Raman shift.

Figures 15 (a) and (b) are measured Raman spectra for Au rhombic nanoparticles array, and cross-star-like nanoparticles array, respectively. It can be seen that the pure spider-web-like nanostructure and the hybrid array have extraordinary Raman enhancement in comparison to the other two Au nanoparticles arrays. The Raman shifts of $611 \mathrm{~cm}^{-1}, 1184 \mathrm{~cm}^{-1}, 1310 \mathrm{~cm}^{-1}$, $1364 \mathrm{~cm}^{-1}$ and $1509 \mathrm{~cm}^{-1}$ can be clearly observed from Fig. 15 (b). It may attribute to the significant contribution from the spider-web-like nanostructure with numerous $\mathrm{Au}$ nanowires and "nano-probes" (the broken wires with sharp tips) whereas the SPPs enhanced E-field and numerous "hot-spots" can be formed. In addition, the rhombic Au particles array with designed "bow-tie"-like apex-to-apex structures in horizontal direction whereas numerous hot-spots can be formed is another reason for the extreme enhanced Raman intensity. 


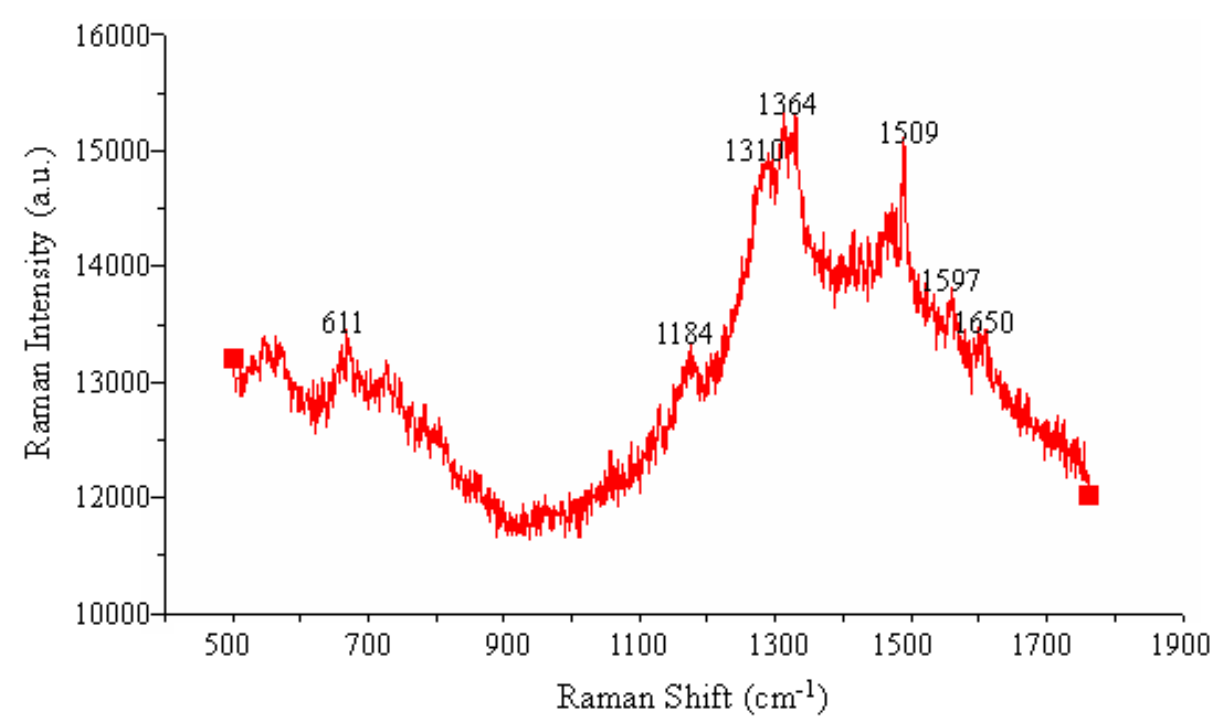

(a)

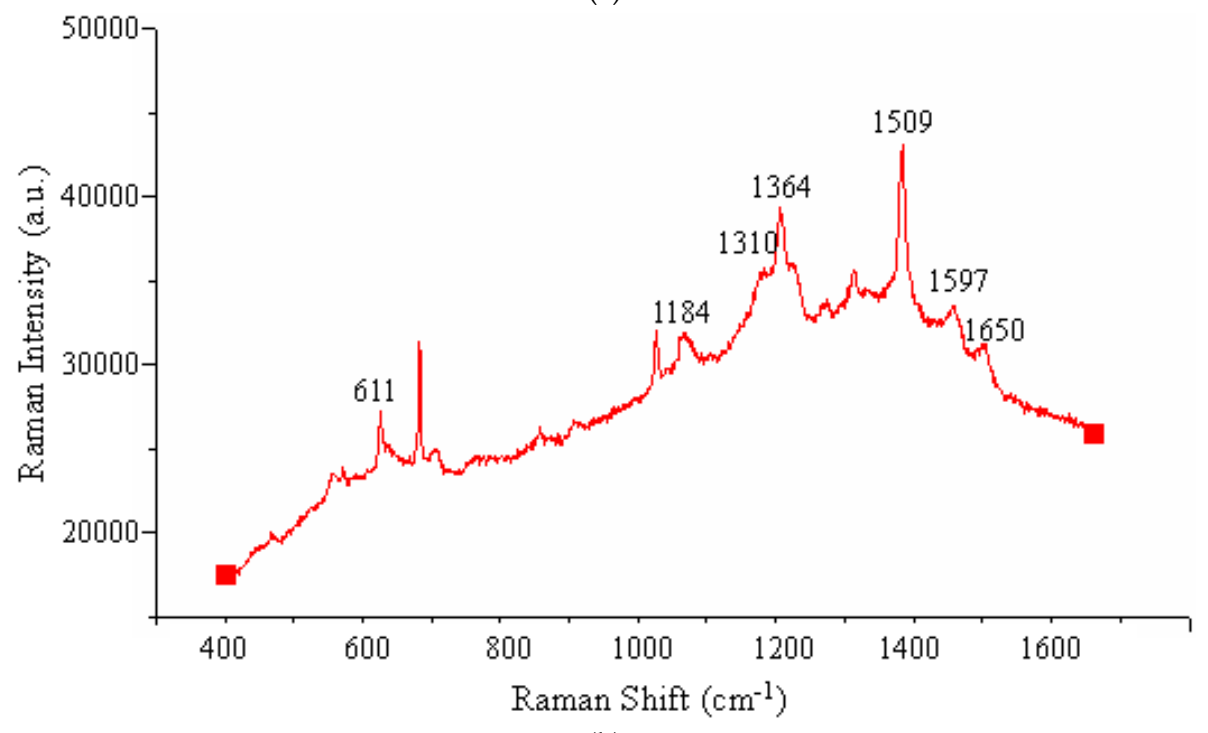

(b)

Fig. 15. Measured Raman spectra for (a) rhombic nanoparticles array (see Fig. 1(b))); (b) hybrid spider-web-like rhombic Au nanoparticles array.

\section{References}

[1] T. W. Ebbesen, H. J. Lezee, H. F. Ghaemi, T. Thio, and P. A. Wolff, Extraordinary optical transmission through subwavelength hole arrays. Nature, 391, 667-669 (1998).

[2] C. Genet and T. W. Ebbesen, Light in tiny holes. Nature, 445, 39-46 (2007). 
[3] E. Laux, C.Genet, and T. W. Ebbesen, Enhanced optical trasmission at the cutoff transition. Opt Express 17, 6920-6930 (2009).

[4] Zhichao Ruan, and Min Qiu, Enhanced Transmission through Periodic Arrays of Subwavelength Holes: The Role of Localized Waveguide Resonances. Phys. Rev. Lett. 96. 233901 (2006).

[5] A. S. Vengurlekar, Extraordinary optical transmission through metal film with subwavelength holes and slits. Current Science, 98, 167-172 (2010).

[6] K. J. Klein Koerkamp, S. Enoch, F. B. Segerink, N. F. Van Hulst, and L. Kuipers, Strong Influence of Hole Shape on Extraordinary Transmission through Periodic Arrays of Subwavelength Holes. Phys. Rev. Lett. 92. 183901 (2004).

[7] Li Hai-jun, Zhang Xiao-dong, Wang Min-rui, Lin Wen-kui, Shi Wen-hua, Zhong Fei, and Zhang Bao-shun, Effects of the periodicity of the subwavelength hole arrays and hole shape of a thin gold film on the optical transmission characteristics. Optoelectron. Lett. 6(3), 78-80 (2010).

[8] A. Degiron and T. W. Ebbesen, The role of localized surface plasmon modes in the enhanced transmission of periodic subwavelength apertures. J. Opt. A: Pure Appl. Opt. 7, S90-S96 (2005).

[9] Andrey K. Sarychev, Viktor A. Podolskiy, A. M. Dykhne, and Vladimir M. Shalaev, Resonance transmittance through a metal film with subwavelength holes. IEEE Journal Of Quantum Electronics, Vol. 38, No. 7, July 2002.

[10] Brian Leathem (BSc. Simon Fraser University, 2001) Thesis-optical transmission through nanohole arrays.Chap.4, p30.

[11] L. Martín-Moreno, F. J. García-Vidal, H. J. Lezec, K. M. Pellerin, T. Thio, J. B. Pendry, and T.W. Ebbesen, Theory of Extraordinary Optical Transmssion through Subwavelength Hole Arrays. Phy. Rev. Lett. 86, 1114 (2001).

[12] Yong-Jun Bao, Ru-Wen Peng, Da-Jun Shu, Mu Wang, Xiang Lu, Jun Shao, Wei Lu, and Nai-Ben Ming, Role of Interference between Localized and Propagating Surface Waves on the Extraordinary Optical Transmission Through a SubwavelengthAperture Array. Phys. Rev. Lett. 101, 087401 (2008).

[13] Rongjing He, Xiuli Zhou, Yongqi Fu, Near Field Optical Experimental Investigation of Gold Nanohole Array, Plasmonics, 6, (2011). (in press) DOI: 10.1007/s11468-0109183-2.

[14] Yongqi Fu, W. Zhou, L.E.N.Lim, C.L. Du, X.G.Luo, Plasmonic microzone plate: Superfocusing at visible regime. Appl. Phys. Lett. 91, 061124 (2007).

[15] Yongqi Fu, Wei Zhou, Lim Enk Ng Lennie, Propagation properties of a plasmonic micro-zone plate in near-field region. J. Opt. Soc. Amer. A, 25, 238-249 (2008).

[16] Yongqi Fu, Yu Liu, Xiuli Zhou, Shaoli Zhu, Experimental demonstration of focusing and lasing of plasmonic lens with chirped circular slits, Opt. Express 18 (4), 34383443 (2010).

[17] Yongqi Fu, Xiuli Zhou, Yu Liu, Ultra-enhanced lasing effect of plasmonic lens structured with elliptical nano-pinholes distributed in variant period. Plasmonics 5(2), 111-116 (2010).

[18] Yiwei Zhang, Yongqi Fu, Yu Liu, Xiuli Zhou, Experimental study of metallic elliptical nanopinhole structure-based plasmonic lenses. Plasmonics, 5(4), (2010). (in press) DOI: 10.1007/s11468-010-9191-2. 
[19] Haiying Li, Xiangang Luo, Chunlei Du, Xunan Chen, and Yongqi Fu, Ag particles array fabricated using laser interference technique for biosensing, Sensors and Actuators B Chemical 134, 940-944 (2008).

[20] Cees J. M. van Rijn, Laser interference as a lithographic nanopatterning tool, J. Microlith., Microfab., Microsyst. 5(1), 121-126( 2006).

[21] Shaoli Zhu, Fei Li, Chunlei Du, Yongqi Fu, A novel bio-nanochip based on localized surface plasmon resonance spectroscopy of rhombic nanoparticles, Nanomedicine Vol. 3, No. 5, 669-677 (October 2008). 


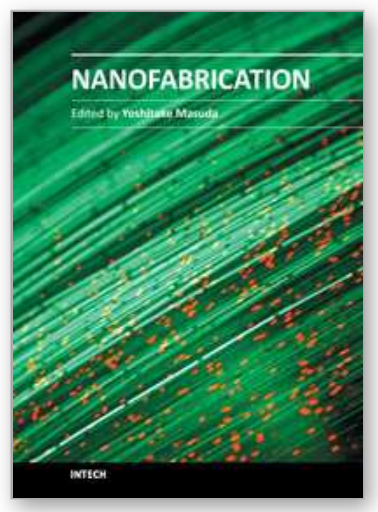

\author{
Nanofabrication \\ Edited by Dr. Yoshitake Masuda
}

ISBN 978-953-307-912-7

Hard cover, 354 pages

Publisher InTech

Published online 22, December, 2011

Published in print edition December, 2011

We face many challenges in the 21st century, such as sustainably meeting the world's growing demand for energy and consumer goods. I believe that new developments in science and technology will help solve many of these problems. Nanofabrication is one of the keys to the development of novel materials, devices and systems. Precise control of nanomaterials, nanostructures, nanodevices and their performances is essential for future innovations in technology. The book "Nanofabrication" provides the latest research developments in nanofabrication of organic and inorganic materials, biomaterials and hybrid materials. I hope that "Nanofabrication" will contribute to creating a brighter future for the next generation.

\title{
How to reference
}

In order to correctly reference this scholarly work, feel free to copy and paste the following:

Yongqi Fu, Fengzhou Fang and Zongwei Xu (2011). Nanofabrication and Characterization of Plasmonic Structures, Nanofabrication, Dr. Yoshitake Masuda (Ed.), ISBN: 978-953-307-912-7, InTech, Available from: http://www.intechopen.com/books/nanofabrication/nanofabrication-and-characterization-of-plasmonicstructures

\section{INTECH}

open science | open minds

\section{InTech Europe}

University Campus STeP Ri Slavka Krautzeka 83/A 51000 Rijeka, Croatia Phone: +385 (51) 770447

Fax: +385 (51) 686166 www.intechopen.com

\section{InTech China}

Unit 405, Office Block, Hotel Equatorial Shanghai No.65, Yan An Road (West), Shanghai, 200040, China 中国上海市延安西路65号上海国际贵都大饭店办公楼405单元 Phone: +86-21-62489820

Fax: +86-21-62489821 
(C) 2011 The Author(s). Licensee IntechOpen. This is an open access article distributed under the terms of the Creative Commons Attribution 3.0 License, which permits unrestricted use, distribution, and reproduction in any medium, provided the original work is properly cited. 